И.Ю. Чернявский${ }^{1}$, С.Ю. Петрухин ${ }^{1}$, В.Б. Матыкин ${ }^{1}$, В.Е. Гайдабука ${ }^{1}$, В.В. Тютюник ${ }^{2}$

${ }^{1}$ Военный институт танковых войск НТУ «ХПИ», Харьков

${ }^{2}$ Национальный университет гражданской защить Украины, Харьков

\title{
ИНФОРМАЦИОННО-ЛОГИЧЕСКАЯ МОДЕЛЬ УЧЁТА НЕОБХОДИМЫХ И ДОСТАТОЧНЫХ ПАРАМЕТРОВ ДЛЯ ОЦЕНКИ ДОЗЫ ГАММА-НЕЙТРОННОГО ИЗЛУЧЕНИЯ ПРОНИКАЮЩЕЙ РАДИАЦИИ
}

В работе анализируются закономерности в оценке полей гамма-нейтронного излучения проникающей радиации боеприпасов различного типа, необходимые и достаточные параметры для корректной оченки доз гамма-нейтронного излучения. На основе использования функционально-структурного подхода, при котором сетевая структура оценки полей гамма-нейтронного излучения формируется иеленаправленно, описывая предметное пространство в виде системы логических уравнений на языке алгебры предикатов, разработана информационно-логическая модель, которая может быть использована в системах радиационного мониторинга.

Ключевые слова: информационно-логическая модель, функиионально-структурный подход, гамма-нейтронное излучение проникающей радиачии, тип боеприпаса.

\section{Введение}

Постановка проблемы. Для каждой мощности и вида ядерного взрыва, а для боеприпасов малого и сверхмалого калибров - и типа боеприпаса, существует определённая закономерность изменения вероятности определённой степени радиационного поражения в зависимости от расстояния $[1,2]$. Это дает возможность, используя приведённые зоны поражений, с приемлемой для ручных (штабных) расчётов точностью, оценивать тяжесть ядерных ударов, определять границы очагов как комбинированных, так и поражений «в чистом виде», пользуясь заранее составленными сравнительно простыми для практики зависимостями, графиками, таблицами.

С другой стороны, существуют полуэмпирические модели как в зарубежной $[3,4]$ так и в отечественной литературе [2, 5-10], полученные в результате полигонных испытаний и позволяющие рассчитывать основные параметры полей гамма-нейтронного излучений в любой точке пространства. Данные выражения с учётом многочисленных поправочных коэффициентов для каждого из типов боеприпасов не нашли своего применения, на наш взгляд, из-за неприемлемой точности существующих на тот момент времени методов и способов определения параметров ядерного взрыва, параметров среды, а также низкого уровня вычислительной техники. Основной закономерностью решения неопределённости при таком подходе является зависимость величины интервала, вмещающего истинное значение об этом параметре, от объема информации. Чем меньше информации, тем больше интервал и роль субъективного фактора. В случае полной информации интервал превращается в точное значение. Общие вопросы определения критерия оптимальности объема необходимой и достаточной информации - соотношения её количества и ценности, - были широко исследованы в работах Горского Ю.М. [28], заложившего основы системноинформационного анализа процессов управления.

На наш взгляд данные вопросы актуальны и для задачи корректной оценки дозовых полей гамманейтронного излучения проникающей радиации боеприпасов различного типа. Создание адекватных моделей в оценки полей гамма-нейтронного излучения может способствовать совершенствованию расчётного метода прогнозирования результатов поражений [1215], а также созданию автоматизированных систем радиационного мониторинга, оценки ситуаций и принятия оптимальных управленческих решений. Основными вопросами при создании таких моделей при оценки доз гамма-нейтронного излучения проникающей радиации (ПР), на наш взгляд, являются:

- достоверный учёт параметров ядерного взрыва боеприпасов сверхмалого и малого калибра. Данные вопросы нами были рассмотрены в работе [11];

- определения типа ядерного боеприпаса, определяемые по спектральным характеристикам нейтронного излучения. В работе [21] нами показано, что неучёт типа ядерного боеприпаса, при оценках ядерного поражения в современных условиях может привести к значительным (недопустимым) ошибкам в оценках тяжести поражения (рис. 1).

В работе Ю.Н. Тарасенко [7] обосновываются необходимые и достаточные для корректной оценки полей гамма-нейтронного излучения характеристики ПР, создаваемые при взрывах боеприпасов различного типа. Важнейшими из них являются: спектральное распределение, амплитудно-временные параметры гамма-нейтронного излучения, накопление дозы во времени и пространственно-угловое распределение ионизирующих излучений; 


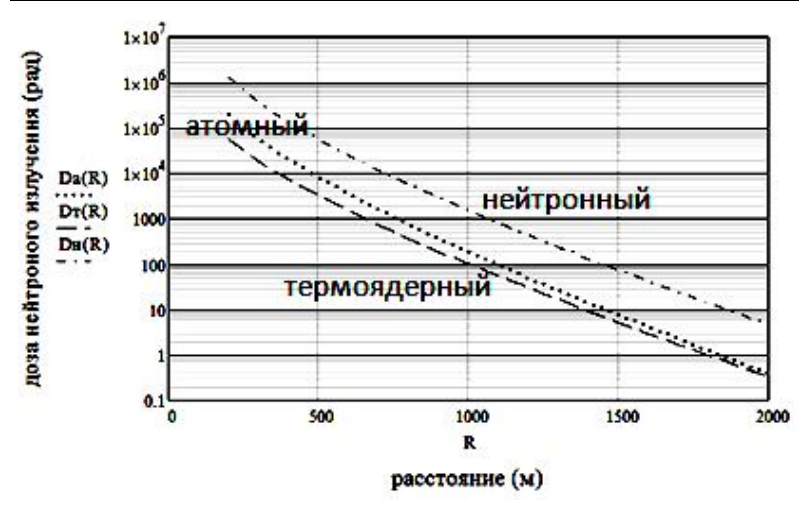

Рис.1. Зависимости дозы нейтронной компоненты проникающей радиации от расстояния до центра взрыва мощностью 1 кт для различных типов

боеприпаса при низком воздушном ЯВ

- корректное описания функций прохождения гамма-нейтронного излучения в воздухе, зависящие как от энергии излучений, так и от параметров среды между центром взрыва и объектом. Это связано с тем, что в воздухе важную роль играют сезонные и суточные изменения плотности и, в меньшей степени, изменения влажности. Как правило, это является заметным источником ошибок вычисления доз гамма-нейтронного излучения ПР. В грунте наиболее значимое влияние на распространение нейтронов оказывает изменения влажности. Для больших расстояний от центра взрыва основную неопределённость в результаты оценки дозы проникающей радиации вносит погрешность всевозможных констант взаимодействия нейтронов и гамма-квантов определённой энергии с веществом. Наиболее полно данные вопросы рассмотрены в работах [16-20].

Снижение погрешности математических моделей, позволяющих корректно оценивать дозы гамманейтронного излучения, возможно за счёт структурных объединений и обязательной отработки следующих основных этапов[19]:

- выбор наиболее перспективных математических методов и создание на их основе математических моделей и алгоритмов, позволяющих эффективно воспроизводить прохождение излучения в веществе при минимальных допущениях относительно процессов взаимодействия и геометрии задачи;

- создание (обоснованный выбор) библиотеки константного обеспечения, позволяющей проводить расчёты с необходимой точностью и детализацией.

В работах $[19,20]$ отмечается, что необходимость рассмотрения высокой степени детализации характеристик гамма-нейтронного излучения ПР с учётом нестационарности процесса и реальной геометрии иногда вступает в противоречие с возможностями ЭВМ. Добиться существенного сокращения расчётного времени, как правило, можно лишь учитывая конкретные особенности геометрии решаемой задачи и возможности использования имеющейся априорной информации, т.е создания специализиро- ванных баз знаний для оценки полей проникающей радиации боеприпасов различного типа.

Повысить эффективность функционирования таких специализированных баз знаний в системе радиационного мониторинга (выявления и оценки обстановки) целесообразно путём формирования целенаправленных структур за счёт применения функционально-структурного подхода, описанного в [22]. Функции оценки полей гамма-нейтронного излучения целесообразно вначале описать в виде системы логических уравнений на языке алгебры предикатов (АП). Предикат - это любая функция $\mathrm{P}\left(\mathrm{x}_{1}, \mathrm{x}_{2}, \ldots, \mathrm{x}_{\mathrm{m}}\right)$, которая задана в предметном пространстве $\mathrm{S}=\mathrm{A}_{1} \times \mathrm{A}_{2} \times \ldots \times \mathrm{A}_{3}$, которая отображает пространство $\mathrm{S}$ в множестве $\mathrm{B}=\{0,1\}$, где 0 і 1 булевы переменные.

Формально операцию замены отношения предикатом можно записать следующим образом:

$$
\mathrm{P}\left(\mathrm{x}_{1}, \mathrm{x}_{2}, \ldots, \mathrm{x}_{\mathrm{m}}\right)=\left\{\begin{array}{l}
1, \text { если } \mathrm{x}_{1}, \mathrm{x}_{2}, \ldots, \mathrm{x}_{\mathrm{m}} \in \mathrm{Q}, \\
0, \text { если } \mathrm{x}_{1}, \mathrm{x}_{2}, \ldots, \mathrm{x}_{\mathrm{m}} \notin \mathrm{Q},
\end{array}\right.
$$

где Q - отношение, а P - предикат.

Обратный переход от предиката к отношению формально будет записан:

если $\mathrm{P}\left(\mathrm{x}_{1}, \mathrm{x}_{2}, \ldots, \mathrm{x}_{\mathrm{m}}\right)=1$, то $\mathrm{x}_{1}, \mathrm{x}_{2}, \ldots, \mathrm{x}_{\mathrm{m}} \in \mathrm{P}$, если $\mathrm{P}\left(\mathrm{x}_{1}, \mathrm{x}_{2}, \ldots, \mathrm{x}_{\mathrm{m}}\right)=0$, то $\mathrm{x}_{1}, \mathrm{x}_{2}, \ldots, \mathrm{x}_{\mathrm{m}} \notin \mathrm{P}$.

Таким образом, любому отношению $\mathrm{Q}$ во взаимно-однозначное соответствие становится предикат, который на языке алгебры предикатов можно записать в виде формулы, используя базисные предикаты 0,1 , и предикат узнавания предмета $\mathrm{x}_{\mathrm{i}}^{\mathrm{a}}$, a также базисные операции конъюнкции $\wedge$ и дизъюнкции $\vee$.

В итоге, применение математического аппарата алгебры предикатов позволит перейти к единой математической модели информационного обеспечения системы радиационного мониторинга, что, с нашей точки зрения, является актуальным и перспективным направлением в современных условиях.

Цель работы: на основе анализа существующих закономерностей в оценки полей гамма-нейтронного излучения проникающей радиации для боеприпасов различного типа выделить необходимые и достаточные для учёта параметры; построить логические уравнения на языке алгебры предикатов для создания информационно - логической модели оценки полей гамма-нейтронного излучения.

\section{1. Анализ необходимых и достаточных параметров в оценке полей гамма-нейтронного излучения проникающей радиации}

Суммарные дозы проникающей радиации определяются суммой доз $\mathrm{D}_{\gamma}$ и $\mathrm{D}_{\mathrm{n}}$, создаваемых всеми компонентами гамма-нейтронного излучения. По известным выражениям, приведенных в работах 
[9,10,13], на основании математического пакета программ Mathcad нами получены зависимости как суммарной дозы ПР, так и её компонентов, от расстояния до центра взрыва нейтронного боеприпаса мощностью в 1 кт, взорванного на высоте 150 м (рис. 2).

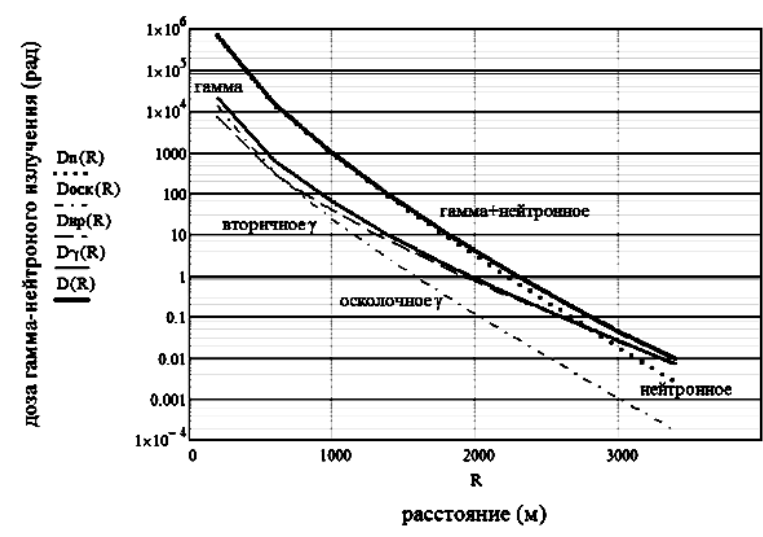

Рис.2. Зависимость суммарной дозы гамма-нейтронного излучения, а также их компонентов от расстояния до центра взрыва

С точки зрения оценки степени тяжести «чистого» радиационного поражения, что характерно для тактических ядерных боеприпасов, для оценки дозовых полей гамма-нейтронного излучения представляет наибольший интерес расстояние на открытой местности от 200 до 3000 м от центра взрыва. Поражающее действие нейтронной компоненты $\mathrm{D}_{\mathrm{n}}$ (рис. 2) в большинстве случаев определяется мгновенными нейтронами, составляющими 99\% общего количества нейтронов. Количество нейтронов, выходящих из зоны взрыва, и их энергетическое распределение зависит от конструкции и типа боеприпаса (рис. 1), что в предложенных зависимостях $[9,10,13]$ не учитывается. Идентификация типа ядерного боеприпаса может заключаться в анализе процентного состава спектра нейтронной компоненты (табл.1). Например, для термоядерного боеприпаса, действующего по принципу деление-синтез-деление, существует три максимума с энергиями 800 кэВ, 4 МэВ и 14 МэВ [23].

Таблица 1

Процентный состав спектра нейтронов (цит. по [7, 16])

\begin{tabular}{|l|l|l|l|}
\hline $\mathrm{E}_{\mathrm{n}}$, МэВ & атомный & термоядерный & нейтронный \\
\hline $14-12,2$ & 0,31 & 5,31 & 40,48 \\
\hline $12,2-10$ & 0,35 & 1,94 & 5,05 \\
\hline $10-8,18$ & 0,23 & 1,28 & 3,31 \\
\hline $8,18-6,3$ & 1,34 & 1,82 & 2,61 \\
\hline $6,3-4$ & 7,13 & 3,28 & 4,32 \\
\hline $4-2,3$ & 6,58 & 6,03 & 7,14 \\
\hline $2,3-1,1$ & 15,38 & 9,03 & 11,88 \\
\hline
\end{tabular}

На рис. 3 представлена разработанная нами блок-схема алгоритма оценки дозовых полей гамманейтронного излучения проникающей радиации ЯВ, учитывающая мощность и тип ядерного боеприпаса.
Пункты 5-9 для термоядерного, 14-17 для атомного и 19-22 для нейтронного боеприпаса осуществляют определение доз гамма-нейтронного излучения с учётом специфики типа ядерного боеприпаса. Для оценки жёсткости нейтронного излучения в специальной литературе $[3,4,9,10]$ введён множитель, отражающий выход нейтронов при взрыве боеприпаса данного типа $\mathrm{K}_{\mathrm{n}}$, лежащий в пределах от $5 \cdot 10^{-9}$ до $43 \cdot 10^{-9}$. Увеличение мощности взрыва нейтронного боеприпаса свыше 10 кт не имеет практического значения из-за поглощения нейтронов в окружающей среде, что было учтено при разработке блок-схемы (рис. 3).

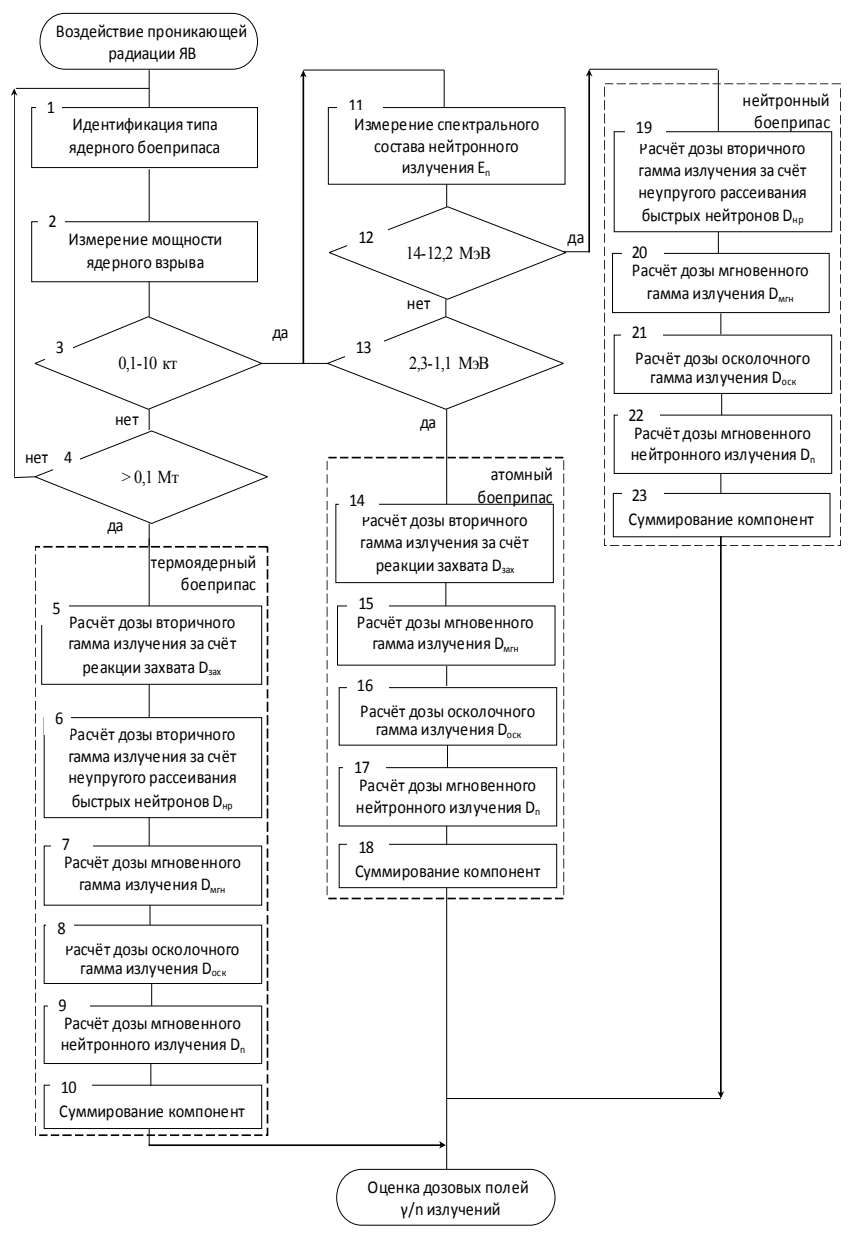

Рис. 3. Блок-схема алгоритма оценки дозовых полей гамма-нейтронного излучения проникающей радиации ЯВ

Процесс распространения нейтронного излучения ПР в воздухе является весьма сложным, и аналитическое решение задачи по определению дозы нейтронов на различных расстояниях представляет большие трудности. Поэтому во многих случаях для инженерных расчётов целесообразно использовать параметры и соотношения, установленные экспериментально. Функция $\mathrm{f}(\mathrm{m})$ отражает прохождение нейтронов в воздухе и зависит от исходного энергетического спектра нейтронов (табл.1) и массы воздуха $\mathrm{m}=\mathrm{R} \rho$ между центром взрыва и объектом: 


$$
f(m)=e^{-R \Delta / \lambda_{n}}-\alpha e^{-R \lambda_{n}^{\prime} / \Delta},
$$

где $\lambda_{\mathrm{n}}$ - эффективная длина ослабления дозы нейтронов (длина свободного пробега) для разных типов боеприпасов, изменяющаяся в пределах от 203 до 220 м;

$\Delta=\rho / \rho_{0}$ - относительная плотность воздуха (летом в большинстве случаев значение $\Delta$ близко к 0,9 , а в зимнее время - к 1,1);

$\lambda_{n}^{\prime}, \alpha-$ коэффициенты аппроксимации, отражающие специфику прохождения нейтронов в ближней зоне.

Для мгновенных нейтронов, как отмечается в работе [19], плотная подстилающая среда обладает существенно большими замедляющими способностями, что ведёт к ускорению процесса накопления во времени рассеянного излучения и обуславливает установление в энергетических и угловых распределениях близкого к равновесному состояния на меньших расстояниях от источника.

Кроме того, это приводит к увеличению до полутора раз дозы нейтронов на близких от источника расстояниях и уменьшению в 5-8 раз на дальних [19]. Характер и степень влияния границы раздела на дозу быстрых нейтронов оценивается коэффициентом влияния подстилающей поверхности:

$$
\mathrm{K}_{3}=\frac{\mathrm{D}_{3}(\mathrm{R}, \mathrm{m}, \mathrm{H}, \mathrm{h})}{\mathrm{D}_{0}(\mathrm{R}, \mathrm{m})},
$$

где $\mathrm{D}_{3}$ и $\mathrm{D}_{0}$ - дозы нейтронов в однородной воздушной среде и у границ раздела воздух-земля соответственно при равенстве линейных и массовых расстояний.

Степень влияния поверхности определяется высотой взрыва Н, высотой положения объекта $\mathrm{h}$ и расстоянием между ними $\mathrm{R}$ (рис. 4), а также содержанием водорода в плотной среде. В литературе допускают независимость влияния переменных, тогда $\mathrm{K}_{3}=\mathrm{K}(\mathrm{R}) \mathrm{K}(\mathrm{H}) \mathrm{K}(\mathrm{h})$.

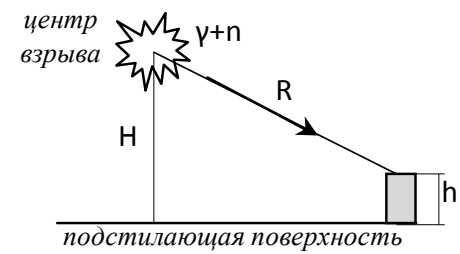

Рис. 4. Влияние поверхности земли на дозу нейтронного излучения

В работе [19] соответствующие зависимости влияния границы раздела воздуха- земля исследованы с помощью строгих моделей и могут быть аппроксимированы следующими соотношениями:

$$
\begin{gathered}
\mathrm{K}(\mathrm{H})=2,4 \cdot\left(1-0,58 \mathrm{e}^{-\mathrm{H} / 250}\right) \cdot \mathrm{K}_{\text {вл }}^{\prime} ; \\
\mathrm{K}(\mathrm{h})=1,44-8,1 /(\mathrm{h}-18,4) ;
\end{gathered}
$$

$$
\mathrm{K}(\mathrm{R})=\left\{\begin{array}{lc}
2,88 \mathrm{R}^{-0,27} \cdot \mathrm{K}_{\text {вл }} \text { для } 50<\mathrm{R} \leq 180 \mathrm{M} ; \\
15 \mathrm{R}^{-0,59} \cdot \mathrm{K}_{\text {вл }} & 180<\mathrm{R} \leq 1500 \mathrm{M} \\
0,2 \cdot \mathrm{K}_{\text {вл }} & \mathrm{R}>1500 \mathrm{M} .
\end{array}\right.
$$

Анализ выражений показывает, что при наземном (контактном) ядерном взрыве $(\mathrm{H}=0) \mathrm{K}(\mathrm{H})=1$ и возрастает с увеличением высоты подрыва ядерного боеприпаса. Для объектов на поверхности земли $\mathrm{K}(\mathrm{h})=1$. Максимальное значение данного коэффициента составляет 1,44 при $\mathrm{h} \geq 200 \mathrm{M}$.

В выражениях (3), (5) используется параметр влажности подстилающей поверхности $\mathrm{K}_{\mathrm{Bл}}^{\prime}\left(\mathrm{K}_{\mathrm{Bл}}\right)$, характеризующий поглощение нейтронного излучения и изменяющийся таким образом: для грунта со средней влажностью до 10\% параметр равен - 1, для грунта повышенной влажности до 20\%-1,05(0,9), для грунта сильной влажности до $50 \%-1,1(0,85)$, для водной поверхности - 1,2(0,6) соответственно. Анализ показывает, что в приземных слоях атмосферы при грунте средней влажности K(R) уменьшается от 0,6 до 0,2 с увеличением расстояния от 200 до 1500 м. На расстояниях более 1500 м $\mathrm{K}(\mathrm{R})=0,2$. Для произвольной влажности грунта коэффициенты влияния расстояния и высоты взрыва рассчитываются по соотношениям [19]:

$$
\begin{gathered}
\mathrm{K}\left(\mathrm{R}, \rho_{\mathrm{H}}\right)=\mathrm{K}(\mathrm{R})\left[1-7 \cdot 10^{-3}\left(10^{-15} \rho_{\mathrm{H}}-9,8\right)\right], \\
\mathrm{K}\left(\mathrm{H}, \rho_{\mathrm{H}}\right)=\mathrm{K}(\mathrm{H})\left[1-1,75 \cdot 10^{-2}\left(10^{-15} \rho_{\mathrm{H}}-9,8\right)\right] \times \\
\times\{1-4[1-0,75 \exp (-\mathrm{H} / 286)] / \mathrm{K}(\mathrm{H})\},
\end{gathered}
$$

где $\rho_{\text {н }}$ - концентрация атомов водорода в подстилающем грунте. Наличие лесных массивов равносильно увеличению влажности грунта и сводится в основном к уменьшению на больших расстояниях дозы нейтронов. Удельный выход мгновенного гамма-излучения сильно зависит от конструктивных особенностей боеприпаса, по оценкам литературных источников, 2/3 квантов имеют энергию от 1 до 4 МэВ (табл. 2).

Таблица 2

Энергетическое распределение мгновенного гамма-излучения в процентах (цит. по $[7,16]$ )

\begin{tabular}{|l|l|l|l|}
\hline $\mathrm{E}_{\gamma}$, МэВ & атомный & термоядерный & нейтронный \\
\hline $4-8$ & 4,4 & 3,6 & 10,3 \\
\hline $2-4$ & 23,6 & 22,4 & 23,5 \\
\hline $2-1$ & 43 & 43 & 33,2 \\
\hline $0,5-1$ & 29 & 31 & 33 \\
\hline
\end{tabular}

На данную компоненту приходится менее 1\% от полной энергии взрыва. Для учёта выхода мгновенных гамма-квантов при взрыве боеприпаса данного типа также, как и для нейтронов, вводится множитель $\mathrm{K}_{\gamma}$ лежащий в пределах от 6,6 до 25 , а также функция, отражающая прохождение излуче- 
ния в воздухе, аналогичная выражению (1). Для мгновенной составляющей гамма-излучения граница раздела воздух - земля может определяться:

$$
\begin{gathered}
\mathrm{K}(\mathrm{H})=1,6 \cdot\left(1-0,375 \mathrm{e}^{-\mathrm{H} / 120}\right) \\
\mathrm{K}(\mathrm{R})= \begin{cases}2,13 \mathrm{R}^{-0,23} & \text { для } 50<\mathrm{R} \leq 500 \mathrm{M} ; \\
0,51 & \mathrm{R}>500 \mathrm{M} .\end{cases}
\end{gathered}
$$

Осколочное гамма-излучение испускается в процессе постепенного радиоактивного распада продуктов деления и, следовательно, его действие в районе взрыва наблюдается вплоть до момента уноса этих продуктов нагретым воздухом в верхние слои атмосферы. Средняя энергия гамма-квантов составляет 2 МэВ. Поскольку осколки деления и продукты их распада представляют собой сложную смесь радиоактивных изотопов, то кинетика испускания ими гамма-излучения имеет весьма сложный характер, количество и энергия гамма-квантов непрерывно изменяются. Поэтому учёт доли энергии взрыва, обусловленной реакцией деления $\eta$ - является обязательным при оценки дозы осколочного гаммаизлучения $\mathrm{D}_{\text {оск }}$ (рис. 2), для различных типов боеприпасов. Кроме того, на формирование поля осколочного гамма-излучения влияют такие физические процессы, как подъём радиоактивного облака и изменение его формы и размеров, вовлечение в облако грунта (создание дополнительного ослабления излучения), отражение и поглощение гамма-квантов поверхностью земли. Большое влияние на распространение гамма-излучения оказывает гидродинамический эффект воздушной полости, связанный с прохождением ударной волны и приводящий к уменьшению поглощения гамма-излучения в десятки раз в зависимости от мощности взрыва. Учет этого фактора для ядерного взрыва мощностью 1-2 Мт приводит к увеличению

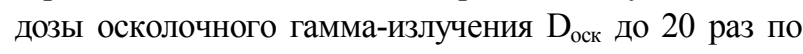
сравнению со случаем невозмущённой атмосферы. Некоторые результаты расчёта с учётом отмеченных особенностей источника излучений и среды их рас- пространения приведены в работе [19]. Вторичное гамма-излучение включает ряд составляющих, из которых основными являются:

- гамма-излучение, возникающее при неупругом рассеивании нейтронов высоких энергий (более 4-6 МэВ) на ядрах атомов воздуха и грунта;

- захватное гамма-излучение, испускаемое при реакциях захвата медленных и тепловых нейтронов ядрами азота воздуха и различных элементов грунта и материала защиты.

Проведённый анализ показал, что вклад вторичных квантов в общую дозу сильно зависит от спектра нейтронов (табл. 1), условий и вида взрыва. Для атомных и термоядерных боеприпасов основной составляющей вторичного гамма-излучения является захватное $\mathrm{D}_{\text {зах }}$, а для нейтронных боеприпасов - гамма-излучения неупругого рассеяния $\mathrm{D}_{\text {нр }}$ (рис. 2). При воздушных и наземных взрывах захватное гамма-излучение «рождается» в зоне взрыва радиусом порядка 300 м.

В работе [20] заявляется, что максимальная мощность дозы вторичного гамма-излучения при энергии быстрых нейтронов в 14 МэВ может до 80 раз превышать соответствующие характеристики для атомного взрыва при равных энерговыделениях. Наличие границы раздела с грунтом приводит к увеличению плотности источников вторичного гаммаизлучения и, как следствие, к увеличению дозы $\mathrm{D}_{\text {нр }}$ на близких расстояниях от центра взрыва. Данное влияние целесообразно описывать соотношением:

$$
K_{3 \gamma}(R)= \begin{cases}0,2+\frac{250}{R+100}, & R \leq 1500 \text { м, } \\ 0,36, & R \geq 1500 \text { м. }\end{cases}
$$

На основании проведенной декомпозиции полуэмпирических зависимостей нами предложена иерархическая структура учёта необходимых и достаточных параметров [25] для корректной оценки полей гамма-нейтронного излучения проникающей радиации (рис. 5).

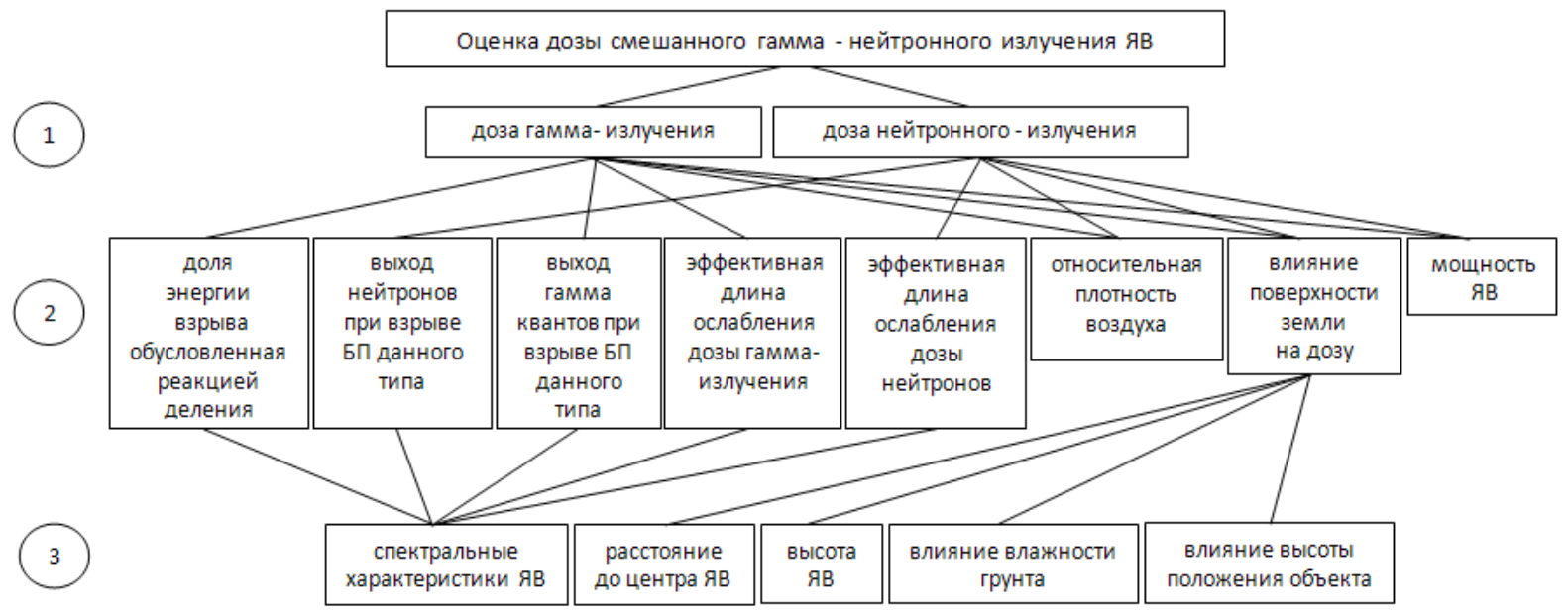

Рис.5. Декомпозиция задачи учёта необходимых и достаточных параметров гамма-нейтронного излучения ПР 
Необходимые и достаточные показатели находятся на разных уровнях иерархии (рис.5), однако могут составлять единую базу данных для оценки гамма-нейтронного излучения.

В полученной нами иерархической структуре целесообразно выделить показатели, характеризующие ядерный боеприпас, и состояния среды (атмосфера, подстилающая поверхность), большая часть которых зависят от спектральных характеристик гамма-нейтронного излучения, a, значит, от типа боеприпаса (рис.1).

Кроме того, полученная иерархическая структура позволяет выделить для дальнейшего анализа более простые составляющие, а попарное сравнение элементов между собой делает возможным численное представление величины влияния каждого элемента иерархии на достижение поставленной цели, т.е точную оценку доз гамма-нейтронного излучения проникающей радиации.

\section{2. Построение логических уравнений на языке алгебры предикатов для создания математической модели оценки полей гамма-нейтронного излучения}

Унивёрсум U рассмотренной нами задачи представлен множеством всех объектов, входящих в базу знаний системы радиационного мониторинга. При переходе от графического изображения конечных предикатов введем бинарный предикат $\mathrm{P}\left(\mathrm{x}_{1}, \mathrm{x}_{2}\right)$, который описывает отношения: 1 , если два элемента $\mathrm{x}_{1}, \mathrm{x}_{2}$ находятся в отношении, и 0 в противоположенном случае. Каждому предикату соответствует дуга иерархической системы, соединяющая вершины, в которых находятся его переменные или их значения. Предложенная нами иерархическая структура информации о поражающих факторах ЯВ представлена на рис. 6.

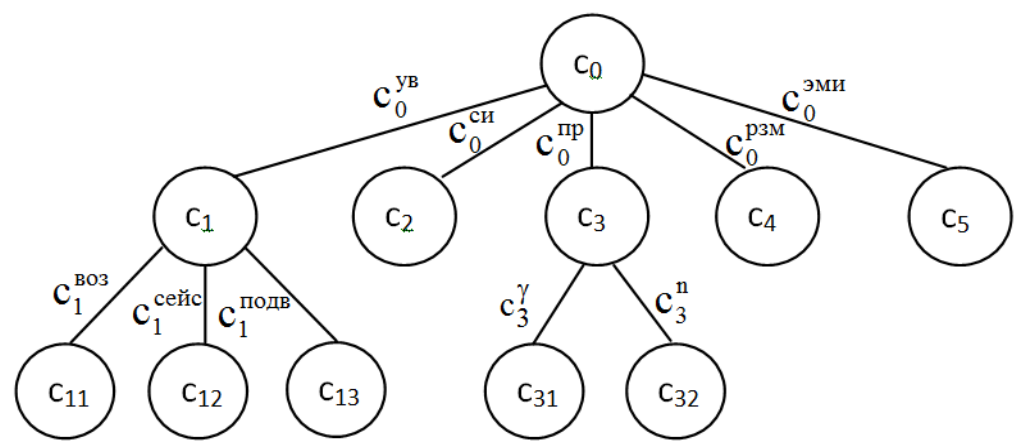

Рис. 6. Иерархическая структура элемента $\mathrm{c}_{0}$ - «поражающие факторы ЯВ»

Декомпозиция информации по поражающим факторам ЯВ языком алгебры конечных предикатов имеет следующий вид:

$$
\mathrm{c}_{0}^{\mathrm{yв}} \vee \mathrm{c}_{0}^{\mathrm{cи}} \vee \mathrm{c}_{0}^{\text {пр }} \vee \mathrm{c}_{0}^{\mathrm{p} 3 \mathrm{M}} \vee \mathrm{c}_{0}^{\text {эми }}=1 .
$$

При этом каждый из поражающих факторов: ударная волна (УВ), световое излучение (СИ), проникающая радиация (ПР), радиоактивное заражение местности (РЗМ) и электромагнитный импульс (ЭМИ) может быть записан как конечный предикат. Область определения переменной для проникающей радиации ( $\mathrm{c}_{0}^{\text {пр }}$ ) описывается уравнением: $\mathrm{c}_{3}^{\gamma} \vee \mathrm{c}_{3}^{\mathrm{n}}=1$ соответственно имеем:

$\gamma$-излучение $\mathrm{P}\left(\mathrm{c}_{0}, \mathrm{c}_{3}, \mathrm{c}_{31}\right)=\mathrm{c}_{0}^{\text {пр }}, \mathrm{c}_{3}^{\gamma}=\Pi \Phi_{1}^{\text {пр }}$;

$n$-излучение $\mathrm{P}\left(\mathrm{c}_{0}, \mathrm{c}_{3}, \mathrm{c}_{32}\right)=\mathrm{c}_{0}^{\Pi p}, \mathrm{c}_{3}^{\mathrm{n}}=\Pi \Phi_{2}^{\text {пр }}$.

Языком алгебры конечных предикатов иерархическую структуру для типов ядерного боеприпаса целесообразно записать:

$$
\mathrm{e}_{0}^{\mathrm{a}} \vee \mathrm{e}_{0}^{\mathrm{t}} \vee \mathrm{e}_{0}^{\mathrm{n}}=1
$$

атомный $\mathrm{P}\left(\mathrm{e}_{0}, \mathrm{e}_{1}\right)=\mathrm{e}_{0}^{\mathrm{a}}=\mathrm{TБ}^{\mathrm{a}}$; термоядерный $\mathrm{P}\left(\mathrm{e}_{0}, \mathrm{e}_{2}\right)=\mathrm{e}_{0}^{\mathrm{t}}=\mathrm{TБ}^{\mathrm{t}}$;

$$
\text { нейтронный } \mathrm{P}\left(\mathrm{e}_{0}, \mathrm{e}_{3}\right)=\mathrm{e}_{0}^{\mathrm{n}}=\text { ТБ}^{\mathrm{n}} \text {. }
$$

С целью решения практических задач опишем предметное пространство, которое представляет собой базу знаний параметров среды, условий прохождения излучения в пространстве.

В соответствии с представленной на рис. 6 иерархической структурой определим показатели для оценки гамма-нейтронного излучения ПР языком алгебры предикатов [22, 26, 27]. Для этого введём предметные изменения и зададим их значения (табл. 3, ПП - предметные переменные).

Область определения группы показателей определяющие дозу гамма-нейтронного излучения проникающей радиации описывается предикатным уравнением:

$$
\Pi Б \vee \Pi C \vee \Pi О \vee \Pi Д=1 \text {. }
$$

В свою очередь, область определения переменных для показателей ПБ, ПС, ПО, ПД описываются следующими уравнениями:

$$
\Pi \mathrm{C}_{\mathrm{R}} \vee \Pi \mathrm{C}_{\mathrm{H}} \vee \Pi \mathrm{C}_{\mathrm{h}} \vee \Pi \mathrm{C}_{\Delta} \vee \Pi \mathrm{C}_{\mathrm{B}}=1 ;
$$


Группы показателей, определяющие дозу гамма-нейтронного излучения проникающей радиации

\begin{tabular}{|c|c|}
\hline Показатели & ПП \\
\hline 1 & 2 \\
\hline $\begin{array}{l}\text { Показатели, характеризующие ядерный боепри- } \\
\text { пас }\end{array}$ & ПБ \\
\hline тротиловый эквивалент & $\Pi \mathrm{b}_{\mathrm{q}}$ \\
\hline $\begin{array}{l}\text { выход гамма-квантов при взрыве атомного бое- } \\
\text { припаса }\end{array}$ & ПБ $\mathrm{\sigma}_{\gamma \mathrm{a}}$ \\
\hline $\begin{array}{l}\text { выход гамма-квантов при взрыве термоядерного } \\
\text { боеприпаса }\end{array}$ & $\Pi Б_{\gamma t}$ \\
\hline $\begin{array}{l}\text { выход гамма-квантов при взрыве нейтронного } \\
\text { боеприпаса }\end{array}$ & $\Pi Б_{\gamma \mathrm{n}}$ \\
\hline выход нейтронов при взрыве атомного боеприпаса & $\Pi Б_{\text {na }}$ \\
\hline $\begin{array}{l}\text { выход нейтронов при взрыве термоядерного } \\
\text { боеприпаса }\end{array}$ & $\Pi Б_{n t}$ \\
\hline $\begin{array}{l}\text { выход нейтронов при взрыве нейтронного бое- } \\
\text { припаса }\end{array}$ & ПБ $Б_{\mathrm{nn}}$ \\
\hline $\begin{array}{l}\text { доля энергии взрыва, обусловленная реакцией } \\
\text { деления }\end{array}$ & $\Pi Б_{\eta}$ \\
\hline Показатели, характеризующие среду & $\Pi \mathrm{C}$ \\
\hline расстояние от центра взрыва & $\Pi C_{\mathrm{R}}$ \\
\hline высота взрыва & $\Pi \mathrm{C}_{\mathrm{H}}$ \\
\hline высота положения объекта & $\Pi \mathrm{C}_{\mathrm{h}}$ \\
\hline относительная плотность воздуха & $\Pi \mathrm{C}_{\Delta}$ \\
\hline влажность подстилающей поверхности (грунта) & $\Pi \mathrm{C}_{\mathrm{B}}$ \\
\hline $\begin{array}{l}\text { Показатели, характеризующие прохождение } \\
\text { излучения в воздухе }\end{array}$ & ПО \\
\hline $\begin{array}{l}\text { эффективная длина ослабления дозы гамма- } \\
\text { излучения атомного боеприпаса }\end{array}$ & $\Pi \mathrm{O}_{\gamma а}^{\text {эдо }}$ \\
\hline $\begin{array}{l}\text { эффективная длина ослабления дозы гамма- } \\
\text { излучения термоядерного боеприпаса }\end{array}$ & $\Pi \mathrm{O}_{\gamma \mathrm{t}}^{\text {эдо }}$ \\
\hline $\begin{array}{l}\text { эффективная длина ослабления дозы гамма- } \\
\text { излучения нейтронного боеприпаса }\end{array}$ & $\Pi \mathrm{O}_{\gamma \mathrm{n}}^{\text {эдо }}$ \\
\hline $\begin{array}{l}\text { эффективная длина ослабления дозы нейтронов } \\
\text { атомного боеприпаса }\end{array}$ & ПО эда \\
\hline $\begin{array}{l}\text { эффективная длина ослабления дозы нейтронов } \\
\text { термоядерного боеприпаса }\end{array}$ & $\Pi \mathrm{O}_{\mathrm{nt}}^{\text {эдо }}$ \\
\hline $\begin{array}{l}\text { эффективная длина ослабления дозы нейтронов } \\
\text { нейтронного боеприпаса }\end{array}$ & ПО эдо \\
\hline $\begin{array}{l}\text { коэффициенты аппроксимации, отражающие } \\
\text { специфику прохождения гамма-излучения в } \\
\text { ближней зоне для атомного боеприпаса }\end{array}$ & $\Pi \mathrm{O}_{\gamma \mathrm{a}}^{\mathrm{\kappa a}}$ \\
\hline $\begin{array}{l}\text { коэффициенты аппроксимации, отражающие } \\
\text { специфику прохождения гамма-излучения в } \\
\text { ближней зоне для термоядерного боеприпаса }\end{array}$ & $\Pi \mathrm{O}_{\gamma \mathrm{t}}^{\mathrm{Ka}}$ \\
\hline $\begin{array}{l}\text { коэффициенты аппроксимации, отражающие } \\
\text { специфику прохождения гамма-излучения в } \\
\text { ближней зоне для нейтронного боеприпаса }\end{array}$ & $\Pi \mathrm{O}_{\gamma \mathrm{n}}^{\mathrm{\kappa a}}$ \\
\hline $\begin{array}{l}\text { коэффициенты аппроксимации, отражающие } \\
\text { специфику прохождения нейтронов в ближней } \\
\text { зоне для атомного боеприпаса }\end{array}$ & $\Pi O_{\text {na }}^{\text {ка }}$ \\
\hline $\begin{array}{l}\text { коэффициенты аппроксимации, отражающие } \\
\text { специфику прохождения нейтронов в ближней } \\
\text { зоне для термоядерного боеприпаса }\end{array}$ & $\Pi \mathrm{N}_{\mathrm{nt}}^{\mathrm{Ka}}$ \\
\hline $\begin{array}{l}\text { коэффициенты аппроксимации, отражающие } \\
\text { специфику прохождения нейтронов в ближней } \\
\text { зоне для нейтронного боеприпаса }\end{array}$ & $\Pi \mathrm{O}_{\mathrm{nn}}^{\mathrm{Ka}}$ \\
\hline $\begin{array}{l}\text { Показатели характеризующие дозу гамма- } \\
\text { нейтронного излучения проникающей радиации }\end{array}$ & ПД \\
\hline
\end{tabular}

\begin{tabular}{|c|c|}
\hline доза мгновенного гамма-излучения & ПД \\
\hline доза захватного гамма-излучения & пд $д_{\gamma}^{3 a x}$ \\
\hline $\begin{array}{l}\text { доза гамма-излучения за счёт неупругого рас- } \\
\text { сеивания }\end{array}$ & Пд $д_{\gamma}^{\mathrm{Hp}}$ \\
\hline доза осколочного гамма-излучения & Пд оск \\
\hline доза мгновенного нейтронного излучения & ПД \\
\hline
\end{tabular}

$$
\begin{gathered}
\Pi Б_{\mathrm{q}} \vee \Pi Б_{\gamma \mathrm{a}} \vee \Pi Б_{\gamma \mathrm{t}} \vee \Pi Б_{\gamma \mathrm{n}} \vee \Pi Б_{\mathrm{na}} \vee \\
\vee \Pi Б_{\mathrm{nt}} \vee \Pi Б_{\mathrm{nn}} \vee \Pi Б_{\eta}=1 ;
\end{gathered}
$$

$\Pi \mathrm{O}_{\gamma \mathrm{a}}^{\text {эдо }} \vee \Pi \mathrm{O}_{\gamma \mathrm{t}}^{\text {эдо }} \vee \Pi \mathrm{O}_{\gamma \mathrm{n}}^{\text {эдо }} \vee \Pi \mathrm{O}_{\mathrm{na}}^{\text {эдо }} \vee \Pi \mathrm{O}_{\mathrm{nt}}^{\text {эдо }} \vee \Pi \mathrm{O}_{\mathrm{nn}}^{\text {эдо }} \vee$

$$
\vee \Pi O_{\gamma \mathrm{a}}^{\text {ка }} \vee \Pi O_{\gamma \mathrm{t}}^{\text {ка }} \vee \Pi O_{\gamma \mathrm{n}}^{\text {ка }} \vee \Pi O_{\text {na }}^{\text {ка }} \vee \Pi O_{\mathrm{nt}}^{\text {ка }} \vee \Pi O_{\mathrm{nn}}^{\text {ка }}=1 \text {; }
$$

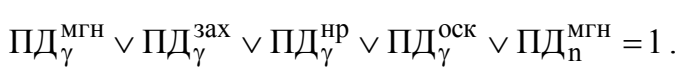

Следует заметить, что при описании таких показателей, как выход гамма квантов при взрыве атомного боеприпаса (ПБ бa $_{\mathrm{a}}$ ), необходимо учитывать как вид излучения, так и тип ядерного боеприпаса. Языком алгебры предикатов это будет записано с помощью импликативного уравнения:

$$
\text { ПБ }{ }_{\gamma \mathrm{a}} \supset \Pi \Phi_{1}^{\Pi \mathrm{p}} \mathrm{TБ}^{\mathrm{a}} .
$$

С учётом этого запишем импликативные уравнения для показателей ПБ ${ }_{\gamma t}, \Pi_{\gamma \mathrm{n}}, \Pi_{\mathrm{na}}, \Pi Б_{\mathrm{nt}}, \Pi_{\mathrm{nn}}$, соответственно:

$$
\begin{aligned}
& \Pi Б_{\gamma \mathrm{t}} \supset \Pi \Phi_{1}^{\Pi \mathrm{p}} \mathrm{TБ}^{\mathrm{t}} ; \\
& \Pi Б_{\gamma \mathrm{n}} \supset \Pi \Phi_{1}^{\Pi \mathrm{p}} \mathrm{TБ}^{\mathrm{n}} \text {; } \\
& \Pi Б_{\mathrm{na}} \supset \Pi \Phi_{2}^{\Pi \mathrm{p}} \mathrm{TБ}^{\mathrm{a}} \text {; } \\
& \Pi Б_{\mathrm{nt}} \supset \Pi_{2}^{\Pi \mathrm{p}} \mathrm{TБ}^{\mathrm{t}} \text {; } \\
& \Pi Б_{\mathrm{nn}} \supset \Pi \Phi_{2}^{\Pi \mathrm{p}} \mathrm{TБ}^{\mathrm{n}} .
\end{aligned}
$$

Запишем предикатное уравнение для области определения переменной ПД:

$$
\Pi Д_{\gamma}^{\mathrm{мгн}} \wedge\left(\Pi_{\gamma}^{3 \mathrm{ax}} \vee \Pi Д_{\gamma}^{\mathrm{\mu p}}\right) \wedge \Pi Д_{\gamma}^{\mathrm{oc \kappa}} \wedge \Pi_{\mathrm{n}}^{\mathrm{M} \Gamma \mathrm{H}}=1
$$

Импликативное уравнение для получения необходимой и достаточной информации по показателям, характеризующим дозу нейтронного излучения проникающей радиации (ПД $\mathrm{n}$ (н) для возможных типов боеприпасов, имеет следующий вид:

$$
\begin{aligned}
& \text { ПД } \mathrm{n} \text { мгн } \supset \\
& \supset \Pi Б_{\mathrm{q}}\left(\Pi Б_{\mathrm{na}} \vee \Pi Б_{\mathrm{nt}} \vee \Pi \mathrm{5}_{\mathrm{nH}}\right) \Pi \mathrm{C}_{\mathrm{R}} \Pi \mathrm{C}_{\mathrm{H}} \Pi \mathrm{C}_{\mathrm{h}} \Pi \mathrm{C}_{\Delta} \Pi \mathrm{C}_{\mathrm{B}} \\
& \left(\Pi \mathrm{O}_{\mathrm{na}}^{\text {эдо }} \vee \Pi \mathrm{O}_{\mathrm{nt}}^{\text {эдо }} \vee \Pi \mathrm{O}_{\mathrm{nn}}^{\text {эдо }}\right)\left(\Pi \mathrm{O}_{\mathrm{na}}^{\text {ка }} \vee \Pi \mathrm{O}_{\mathrm{nt}}^{\text {ка }} \vee \Pi \mathrm{O}_{\mathrm{nn}}^{\text {ка }}\right) \text {, }
\end{aligned}
$$

из которого выходит, что для характеристики дозы нейтронного излучения проникающей радиации $\mathrm{D}_{\mathrm{n}}$ (рис. 2) необходимо иметь следующие выходные данные: тротиловый эквивалент (мощность ядерно- 
го взрыва), выход нейтронов (в зависимости от типа боеприпаса (ПБ центра ядерного взрыва ПС $\mathrm{R}_{\mathrm{R}}$ до объекта, высоту взрыва ПС ностью ПС $\mathrm{h}$, относительную плотность воздуха ПС ${ }_{\Delta}$, влажность подстилающей поверхности (грунта) $\Pi_{\mathrm{B}}$, эффективную длину ослабления дозы нейтронов и коэффициенты аппроксимации, отражающие специфику прохождения нейтронов в ближней зоне в зависимости от типа ядерного боеприпаса.

Аналогично запишем импликативное уравнение для получения необходимой и достаточной информации по показателям:

- характеризующие дозу мгновенного гаммаизлучения проникающей радиации $\mathrm{D}_{\text {мгн }}$ (рис.2) для возможных типов боеприпасов:

$$
\begin{aligned}
& \Pi Д_{\gamma}^{\mathrm{M \Gamma H}} \supset \Pi_{\mathrm{q}}\left(\Pi Б_{\gamma \mathrm{a}} \vee \Pi Б_{\gamma \mathrm{T}} \vee \Pi Б_{\gamma \mathrm{H}}\right) \Pi \mathrm{C}_{\mathrm{R}} \Pi \mathrm{C}_{\mathrm{H}} \Pi \mathrm{C}_{\Delta} \\
& \left(\Pi \mathrm{O}_{\gamma \mathrm{a}}^{\text {эдо }} \vee \Pi_{\gamma \mathrm{t}}^{\text {эдо }} \vee \Pi_{\gamma \mathrm{n}}^{\text {эдо }}\right)\left(\Pi \mathrm{O}_{\gamma \mathrm{a}}^{\text {ка }} \vee \Pi \mathrm{O}_{\gamma \mathrm{t}}^{\text {ка }} \vee \Pi \mathrm{O}_{\gamma \mathrm{n}}^{\text {ка }}\right) \text {. }
\end{aligned}
$$

- характеризующие дозу вторичного гаммаизлучения проникающей радиации за счет неупругого рассеивания быстрых нейтронов $\mathrm{D}_{\text {нр }}$ (рис. 2):

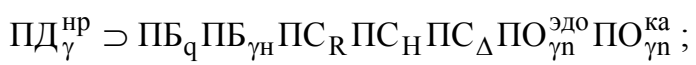

- характеризующие дозу вторичного гаммаизлучения проникающей радиации за счет захвата медленных (тепловых) нейтронов $\mathrm{D}_{3 \mathrm{ax}}$ (рис. 2):

$$
\begin{gathered}
\Pi Д_{\gamma}^{3 а x} \supset \\
\supset \Pi_{\mathrm{q}}\left(\Pi \mathrm{b}_{\gamma \mathrm{a}} \vee \Pi \overline{6}_{\gamma \mathrm{T}}\right) \Pi \mathrm{C}_{\mathrm{R}} \Pi \mathrm{C}_{\mathrm{H}} \Pi \mathrm{C}_{\Delta}\left(\Pi \mathrm{O}_{\gamma \mathrm{a}}^{\text {эдо }} \vee \Pi \mathrm{O}_{\gamma \mathrm{t}}^{\text {эдо }}\right) .
\end{gathered}
$$

- характеризующие дозу осколочного гаммаизлучения проникающей радиации $\mathrm{D}_{\text {оск }}$ (рис. 2):

$$
\begin{gathered}
\text { ПД } \text { оск }_{\gamma} \\
\supset \Pi_{\mathrm{q}} \Pi Б_{\eta} \Pi \mathrm{C}_{\mathrm{R}} \Pi \mathrm{C}_{\mathrm{H}} \Pi \mathrm{C}_{\Delta}\left(\Pi \mathrm{O}_{\gamma \mathrm{a}}^{\text {эдо }} \vee \Pi_{\gamma \mathrm{t}}^{\text {эдо }} \vee \Pi_{\gamma \mathrm{n}}^{\text {эдо }}\right) .
\end{gathered}
$$

Таким образом, разработка алгебры предикатов даёт возможность перехода от алгоритмического описания информационных процессов к описанию их с помощью уравнений, которым не свойственна направленность действия. Важным достоинством метода описания объекта с помощью уравнений является то, что по уравнениям можно определить реакцию объекта при любом способе распределения сигналов между его полюсами.

\section{Выводы}

1. В работе проведен анализ необходимых и достаточных параметров в оценки полей гамманейтронного излучения проникающей радиации. Несмотря на существенные особенности для боеприпасов различного типа данную информацию целесообразно сгруппировать в параметры, характеризующие ядерный боеприпас, характеризующие прохождение излучения в воздухе и состояния среды. Большая часть параметров напрямую зависят от спектральных характеристик гамма-нейтронного излучения, a, значит, от типа ядерного боеприпаса. Предложена блок-схема алгоритма оценки дозовых полей гамма-нейтронного излучения проникающей радиации ЯВ, учитывающая мощность и тип ядерного боеприпаса.

2. На основании проведенной декомпозиции полуэмпирических зависимостей получена иерархическая структура учёта необходимых и достаточных параметров для корректной оценки полей гамманейтронного излучения проникающей радиации.

3. На основе функционально-структурного подхода к построению моделей, описывая предметное пространство в виде системы логических уравнений на языке алгебры предикатов, разработана информационно-логическая модель оценки полей гамма-нейтронного излучения проникающей радиации боеприпасов различного типа. Модель позволяет перейти к единой математической модели комплексной оценки воздействий поражающих факторов боеприпасов различного типа, мощности и вида взрыва.

\section{Список литературы}

1. Зашита от оружия массового поражения / Под.ред. В.В. Мясникова. - 2-е изд., перераб. и доп. -М.: Воениздат, 1989. - 389 c.

2. Защитта объектов народного хозяйства от оружсия массового поражения: Справочник/ Под редакцией Г.П. Демиденко. 2-е изд., перераб. и доп. - К.: Вищашк. Головное изд-во, 1989. -287 с.

3. Действие ядерного взрыва/ Сборник переводов под редакцией С.С. Григоряна и Г. С. Шапиро. -М.: Изд. «Mup», 1971. $-680 \mathrm{c}$.

4. Действие ядерного оружия: Пер. с англ. -М.: Воениздат, 1965. - 683 c.

5. Иванов А.И., Рыбкин Г.И. Поражающее действие ядерного взрыва. -М.: Военное издательство МО Союза ССР, 1960. -378 c.

6. Иванов А.И., Науменко И.А., Павлов М.П. Ракетно-ядерное оружие и его поражающее действие. - М.: Воениздат, 1971. $-224 \mathrm{c}$.

7. Тарасенко Ю. Н. Ионизационные методы дозиметрии высокоинтенсивного ионизирующего излучения М.: Техносфера, 2013. -264 c.

8. Мырова Л.О., Чепиженко А.З. Обеспечение стойкости аппаратуры связи к ионизирующим и электромагнитным излучениям. 2-е изд., перераб. и доп. - М: Радио и связь, 1988. - 296 с.

9. Краснощекова Г.Ф. Особенности проектирования средств специального назначения [Электронный ресурс] Самар. гос. аэрокосм. ун-т им. С.П. Королева (нац. исслед. ун-т); авт.-сост Г.Ф. Краснощекова. - Электрон.текстовые и граф. дан. - Самара, 2012

10. Оченка устойчивости работы электронных систем при воздействии ионизирующих излучений: метод. Пособие для практ. занятий / А.И. Новаша и др. - Минск: БГУИР, 2008. -18 c.

11. Чернявский И.Ю., Григорьев А.Н., Бильк З.В., Матыкин В.Б. Применение кремниевых ріп детекторов для регистрачии параметров ядерного взрыва. Системи озброєння $і$ військова техніка: науковий журнал. $-X$. ХУПС, 2016. - №4(48). - C. 61-68. 
12. Дозовая нагрузка на человека в полях гамманейтронного излучения / Гозенбук В.Л., Кеирим-Маркус И.Б., Савинский А.К., Чернов Е.Н. -М.:, Атомиздат, 1978. -168 c

13. Чернявский И.Ю. Оченка степени радиационного поражения путём прогнозирования дозовых нагрузок за данными датчика боеспособности / И.Ю. Чернявский, B.B. Марущенко, А.В. Матыкин// Системи озброєння $i$ військова техніка: науково - технічний журнал. - $X$ : Харківський університет Повітряних Сил імені Івана Кожедуба, 2016. - №1(45). - С. 125-133.

14. Гаранюшкин Н.В., Кондуршкин Н.А., Шаховский В.В., Шуляк И.П. Алгоритм расчёта полей гамманейтронного излучения (РИНГ-78). Доклад № 7.12 // Вторая Всесоюзная научная конференция по защите от ионизирующих излучений ядерно-технических установок. 19-21 декабря 1978. - М.: МИФИ.

15. Гаранюшкин Н.В., Кондуршкин Н.А. Расчёт захватного гамма-излучения в однородной среде и у границы раздела двух сред на основе комбинирования метода Монте-Карло и полуаналитических методов // Четвёртая Всесоюзная конференция по защите от ионизируюших излучений ядерно- технических установок. Тезисы докл. - Томск: Томский политехнический институт, 1985.- $79 \mathrm{c}$

16. Распространение ионизирующих излучений в воздухе/ Климанов В.А., Коновалов С.А., Кочанов В.А. и др. Под ред В.И. Кухатевича и В.П. Машковича. - М.: Атомиздат, 1979 - $216 \mathrm{c}$.

17. Волощенко А.М., Гончаров А.Н., Кондушкин Н.А. Влияние влажности воздуха на характеристики ионизирующих излучений // Сб. научных трудов МИФИ: Защита от ионизирующих излучений / Под ред. В.К. Сахорова.-М.: Энергоатомиздат, 1988. - C. 57-60.

18. Гаранюшкин Н.В., Кондурушкин Н.А. Банк данных для расчёта полей нейтронов и гамма-излучения от точечных и распределённых источников у границы раздела воздух-земля// Шестая Российская научная конференция по зашите от ионизируюших излучений ядерно-технических установок. Тезисы докладов. Обнинск: ГНЦ ФЭИ, 1994. Т.1. - С. 93-94.

19. Физика ядерного взрыва: Том 1. Развитие взрыва/ Министерство обороны Российской Федерации.
Центральный физико-технический институт. - М.: Наука. Физматлит, 1997. - 528 c

20. Физика ядерного взрыва: Том 2. Действие взрыва/ Министерство обороны Российской Федерации. Центральный физико-технический институт. - М.: Наука. Физматлит, 1997. - 256 с.

21. Чернявский И.Ю. Биологические аспекты импульсного воздействия радиачионного фактора военного характера // Чернявский И.Ю. Марущенко В.В. Гайдабука В.Е., Писарев С.А., Меньшов С.Н., Матикин В.Б. Системи обробки інформації: збірник наук. праць Х.: Харківський університет Повітряних Сил імені Івана Кожедуба, 2016. - Вип.5(142). - C. 193-201.

22. Булкин В.И. Математические модели знаний и их реализация с помощью алгебропредикатных структур / В.И. Булкин, Н.В. Шаронова. - Донеик: Дмитренко Л.Р., 2010. - $304 \mathrm{c}$.

23. Дозиметрия ионизирующих излучений ядерного взрыва: Учебник / Под ред. Шестерякова. - М.: ВАХЗ, 1976. -455 c.

24. Шабанов-Кушнаренко Ю.П. Теория интеллекта. Математические средства. Харьков. Вища школа, 1984. $144 c$.

25. Козуля Т.В., Петрухін С.Ю.Місие екологічного портрету території в інформаційному забезпеченні систем моніторингу // Вестник Херсонского Национального Технического Университета, №4 (27), 2007, - C. 230- 233.

26. Петрухін С.Ю. Напрями розробки інформаційної корпоративної екологічної системи військово- техногенних територій. // Восточно-европейский журнал передовых технологий, 6/5 (42), 2009. - C. 48-52.

27. Петрухін С.Ю.,Артем'єв С.Р., Пісня Л.А. Напрямки вирімення завдань екологічної безпеки військ у миротворчих операчіях // Восточно-европейский журнал передовых технологий, 3/11 (51), 2011. - С. 4-7.

28. Горский Ю.М. Системно- информачионный анализ проиессов управления / Ю.М. Горский. - М., 1988. $322 \mathrm{c}$.

Надійшла до редколегіі 19.02.2018

Рецензент: д-р техн. наук, проф. О.В. Стаховський, Військовий інститут танкових військ НТУ «ХПІ», Харків.

\section{ІНФОРМАЦІЙНО-ЛОГІЧНА МОДЕЛЬ ОБЛІКУ НЕОБХІДНИХ І ДОСТАТНІХ ПАРАМЕТРІВ ДЛЯ ОЦІНКИ ДОЗИ ГАММА-НЕЙТРОННОГО ВИПРОМІНЮВАННЯ ПРОНИКАЮЧОЇ РАДІАЦІї}

І.Ю. Чернявський, С.Ю. Петрухін. В.Б. Матикін, В.С. Гайдабука, В.В. Тютюник

В роботі аналізуються закономірності в очіниі полів гамма нейтронного випромінювання проникаючої радіачії боєприпасів різного типу, необхідні і достатні параметри для коректної оиінки доз гамма-нейтронного випромінювання. На основі використання функціонально-структурного підходу, при якому мережева структура оцінки полів гамма нейтронного випромінювання формується иілеспрямовано, описуючи предметний простір у вигляді системи логічних рівнянь на мові алгебри предикатів, розроблена інформачійно-логічна модель. яка може бути використана в системах радіаційного моніторингу.

Ключові слова: інформаційно-логічна модель, функиіонально-структурний підхід, гамма-нейтронне випромінювання проникаючої радіачії, тип босприпаси.

\section{INFORMATIONAL-LOGICAL MODEL OF ACCOUNTING NECESSARY AND SUFFICIENT PARAMETERS FOR DOSE ASSESSMENT OF GAMMA-NEUTRON RADIATION OF PENETRATING RADIATION}

\section{I.Y. Cherniavskiy, S.Y. Petrukhin. V.B. Matykin, V.E. Gaydabuka, V. V. Tyutyunyk}

This paper analyse regularities in the assessment of the fields of gamma-neutron radiation of penetrating radiation of ammunitions of various types, necessary and sufficient parameters for the correct estimation of doses of gamma-neutron radiation. Based on the use of the functional-structural approach, in which the network structure of the evaluation of gamma-neutron radiation fields is formed purposefully, an information-logical model that can be used in radiation monitoring systems is developed describing the object space in the form of a system of logical equations in the language of predicate algebra.

Keywords: information - logical model, functional - structural approach, gamma - neutron radiation of penetrating radiation, type of ammunition. 